\title{
THE BLOW UP OF THE SOLUTION OF FRACTIONAL ORDINARY DIFFERENTIAL EQUATIONS
}

\section{Bambang Hendriya Guswanto}

Faculty of Sciences and Engineering, Universitas Jenderal Soedirman

Purwokerto, Indonesia

Email : bambanghg_unsoed@yahoo.com

Abstract. This paper discusses the sufficient condition so that the solution of the initial value problem of fractional ordinary differential equation,

$$
\begin{aligned}
D^{\alpha} u(t) & =f(t, u(t)) \text { on }[0, T) \times(0, \infty) . \\
u(0) & =u_{0}>0,
\end{aligned}
$$

where $D^{\alpha}$ is Riemann-Liouville fractional derivative operator defined as

$$
D^{\alpha} u(t):=\frac{1}{\Gamma(1-\alpha)} \frac{d}{d t} \int_{0}^{t}(t-s)^{-\alpha} u(s) d s
$$

and $\Gamma$ is Gamma function with $0<\alpha<1$, exists uniquely and blows up in finite time.

Keywords. fractional ordinary differential equations, existence, uniqueness, blow up.

\section{INTRODUCTION}

Consider the initial value problem of (non-fractional) ordinary differential equation

$$
\begin{aligned}
\frac{d u}{d t} & =f(u), \quad 0<u<\infty . \\
u(0) & =u_{0}>0 .
\end{aligned}
$$

When $f(u)=u^{p}$, the solution of the problem (1) is

$$
u(t)=[(p-1)(T-t)]^{\frac{1}{1-p}}
$$

where $T=\frac{1}{p-1} u_{0}^{1-p}$. If $1<p<\infty$ then $u(t) \rightarrow \infty$ as $t \rightarrow T^{-}$. It means that $u$ can not extend globally in time or $u$ is said to blow up in finite time. Many mathematicians have discussed the blow up of the solution of the (non fractional) ordinary/partial differential equations. One can know the discussion about this in [2] or [3].

In this paper, we will discuss the blow up of the solution of the initial value problem of fractional ordinary differential equation

$$
\begin{aligned}
D^{\alpha} u(t) & =f(t, u(t)) \text { on }(0, T) \times(0, \infty) . \\
u(0) & =u_{0}>0
\end{aligned}
$$


where $D^{\alpha}$ is Riemann-Liouville fractional derivative operator defined as

$$
D^{\alpha} u(t):=\frac{1}{\Gamma(1-\alpha)} \frac{d}{d t} \int_{0}^{t}(t-s)^{-\alpha} u(s) d s
$$

and $\Gamma$ is Gamma function with $0<\alpha<1$. As for fractional differential equations, one can read more completely about it, for an example, in [3]. Specifically, we will find the sufficient condition so that the solution of the problem (2) exists uniquely. Moreover, we will also find the sufficient condition so that the solution blows up in finite time. The result about the blow up notion for fractional ordinary differential (2), as a ordinary diferential equation part of fractional semilinear parabolic equation, will be useful to describe the blow up notion for fractional semilinear parabolic equation itself.

\section{MAIN RESULT}

In order to discuss the blow up notion, of course, we must determine the solution of the problem (2) first. Consider

$$
\frac{1}{\Gamma(1-\alpha)} \frac{d}{d t} \int_{0}^{t}(t-s)^{-\alpha} u(s) d s=f(t, u(t)) .
$$

It follows that

Then we have

$$
\int_{0}^{t}(t-s)^{-\alpha} u(s) d s=\int_{0}^{t} \Gamma(1-\alpha) f(s, u(s)) d s .
$$

$$
u(t)=\Gamma(1-\alpha)(T-t)^{\alpha} f(t, u(t))
$$

for some $T \in(0, \infty)$. By the initial condition,

$$
T=\left(\frac{1}{\Gamma(1-\alpha)} \cdot \frac{u_{0}}{f\left(u_{0}\right)}\right)^{1 / \alpha}
$$

For an example, we will find the solution of the problem (2) for $f(t, u(t))=(u(t))^{p}$. The non trivial solution is

$$
u(t)=\left[\Gamma(1-\alpha)(T-t)^{\alpha}\right]^{\frac{1}{1-p}}
$$

where

$$
T=\left[\frac{1}{\Gamma(1-\alpha)} u_{0}^{1-p}\right]^{1 / \alpha}
$$


Therefore, we have

$$
u(t)=\left[\Gamma(1-\alpha)\left(\left(\frac{u_{0}^{1-p}}{\Gamma(1-\alpha)}\right)^{1 / \alpha}-t\right)^{\alpha}\right]^{\frac{1}{1-p}} .
$$

If $p>1$ then $u(t) \rightarrow \infty$ as $t \rightarrow T^{-}$. It means that the solution $u$ blows up in finite time $T<\infty$. This result is similar to that of the problem of (1), the initial value problem of (non fractional) ordinary differential equation.

Next, we will find the sufficient condition so that the problem (2) has a unique solution. Suppose that $F$ is a function defined as

$$
F(u(t)):=\Gamma(1-\alpha)(T-t)^{\alpha} f(t, u(t))
$$

and there is a function $\mu_{\alpha}$ on $[0, \infty)$ so that $|f(t, u(t))-f(t, v(t))| \leq$ $\mu_{\alpha}(t)|u(t)-v(t)|$ and

$$
0<\mu_{\alpha}(t)<\frac{1}{\Gamma(1-\alpha) T^{\alpha}}, t \in[0, \infty) .
$$

We will investigate the existence and the uniqueness of the solution $u$. Consider

$$
\begin{aligned}
|F(u(t))-F(v(t))| & =\left|\Gamma(1-\alpha)(T-t)^{\alpha}\right||f(t, u(t))-f(t, v(t))| \\
& \leq \Gamma(1-\alpha) T^{\alpha}|f(t, u(t))-f(t, v(t))| \\
& \leq \Gamma(1-\alpha) T^{\alpha} \mu_{\alpha}(t)|u(t)-v(t)| \\
& \leq \rho_{\alpha}(t)|u(t)-v(t)|
\end{aligned}
$$

where $\rho_{\alpha}(t)=\Gamma(1-\alpha) T^{\alpha} \mu(t)$. It is clear that $0<\rho_{\alpha}(t)<1$ based on our assumption. By Contraction Mapping Principle, the solution $u$ exists uniquely.

After discussing the existence and the uniqueness of the solution, we discuss the sufficient condition so that the solution blows up in finite time. Suppose that

$$
f(t, u) \geq 1+\frac{1}{\Gamma(1-\alpha)} \cdot \frac{t u}{T(T-t)^{\alpha}}
$$

on $[0, T) \times(0, \infty)$. It implies

$$
\begin{aligned}
u(t) & =\Gamma(1-\alpha)(T-t)^{\alpha} f(t, u(t)) \\
& \geq \Gamma(1-\alpha)(T-t)^{\alpha}\left(1+\frac{1}{\Gamma(1-\alpha)} \cdot \frac{t u(t)}{T(T-t)^{\alpha}}\right) \\
& \geq \Gamma(1-\alpha)(T-t)^{\alpha}+\frac{t}{T} u(t)
\end{aligned}
$$

or

$$
u(t) \geq \frac{\Gamma(1-\alpha)(T-t)^{\alpha}}{1-\frac{t}{T}} .
$$


Consider that

$$
\begin{aligned}
\lim _{t \rightarrow T^{-}} \frac{\Gamma(1-\alpha)(T-t)^{\alpha}}{1-\frac{t}{T}} & =\lim _{t \rightarrow T^{-}} \frac{\alpha \Gamma(1-\alpha)(T-t)^{\alpha-1}}{\frac{1}{T}} \\
& =\lim _{t \rightarrow T^{-}} \frac{\alpha T \Gamma(1-\alpha)}{(T-t)^{1-\alpha}} \\
& =\infty .
\end{aligned}
$$

Because of (3), we conclude that $\lim _{t \rightarrow T^{-}} u(t)=\infty$. It means that the solution $u$ blows up in finite time $T$.

\section{CONCLUSION}

Based on our discussion in preceding section, we have two main results concerning the blow up of the solution of the problem (2).

The following theorem gives us the sufficient condition so that the problem (2) has a unique solution.

Theorem 1. Suppose that there is a function $\mu_{\alpha}$ on $[0, \infty)$ such that

$$
|f(t, u(t))-f(t, v(t))| \leq \mu_{\alpha}(t)|u(t)-v(t)|
$$

and

$$
0<\mu_{\alpha}(t)<\frac{1}{\Gamma(1-\alpha) T^{\alpha}}, t \in[0, \infty),
$$

where

$$
T=\left(\frac{1}{\Gamma(1-\alpha)} \cdot \frac{u_{0}}{f\left(u_{0}\right)}\right)^{1 / \alpha} .
$$

Then the initial value problem of fractional ordinary differential equation (2) has a unique solution.

The next theorem gives us the sufficient condition so that the solution of the problem (2) blows up in finite time.

Theorem 2. Suppose the function $f:[0, \infty) \times(0, \infty) \rightarrow(0, \infty)$ satisfies

$$
f(t, u) \geq 1+\frac{1}{\Gamma(1-\alpha)} \cdot \frac{t u}{T(T-t)^{\alpha}}
$$

on $[0, T) \times(0, \infty)$. Then the solution of the initial value problem of fractional ordinary differential equation (2) blows up in finite time $T$. 
This result can be used as basis to discuss the blow up of the solution of fractional semilinear parabolic equation. The result about the solution of the linear part of fractional semilinear parabolic equation is also needed to discuss the blow up of the solution of fractional semilinear parabolic equation.

\section{REFERENCES}

[1] Poludbny, I., Fractional Differential Equations, Academic Press, San Diego 1999.

[2] Fila, M., Blow up of Solution of Semilinear Parabolic Equations, Special Lectures at Tohoku University, 2006

[3] Suzuki, T., The Blow Mechanism in Nonlinear Partial Differential Equations - Scaling and Variations, Division of Mathematical Science, Department of Systems Innovation, Graduate School of Engineering Science, Osaka University, 2009. 